\section{Aspectos estruturais e funcionais do apoio social de idosos do Município de São Paulo, Brasil}

\author{
Structural and functional aspects of social support \\ for the elderly in the city of São Paulo, Brazil
}

\footnotetext{
${ }_{1}^{1}$ Instituto de Saúde, Secretaria de Estado da Saúde de São Paulo, São Paulo, Brasil. 2 Faculdade de Saúde Pública, Universidade de São Paulo, São Paulo, Brasil.

Correspondência T. E. C. Rosa Instituto de Saúde, Secretaria de Estado da Saúde de São Paulo. Rua Santo Antonio 590, Bela Vista, $S P$ 01314-000, Brasil. tererosa@isaude.sp.gov.br
}

\section{Abstract}

This study aimed to describe the distribution of social support networks for the elderly in the City of São Paulo, Brazil, based on socioeconomic and demographic characteristics. A probability sample of 1,568 elderly persons ( $\geq 60$ years) was studied (SABE query), focusing on structural and functional dimensions of social networks. Univariate and multiple logistic regression models were used to analyze the data. The best conditions in social networks were in the lowest income levels and the worst among older and unmarried people. For women, unmarried marital status was significantly associated with lower schooling. Cohabiting had the strongest influence on the functional dimension, while "living alone" significantly decreased the odds of social exchange. Multiple logistic regression by gender suggested that age, marital status, per capita income, and schooling were associated with social networks among elderly people. However, these relationships were expressed unevenly between the genders and depending on the socio-demographic characteristics of the different dimensions of the social support network.

Social Support; Aged; Social Conditions
Tereza Etsuko da Costa Rosa 1 Maria Helena D’Aquino Benício 2 Maria Cecília Goi Porto Alves 1 Maria Lúcia Lebrão 2

\section{Introdução}

A partir do final dos anos 70 do século XX, a categoria apoio social tem favorecido o desenvolvimento de diversos trabalhos de investigação que buscam explicar algumas das diferenças existentes na distribuição de certas enfermidades, tanto físicas quanto mentais.

Não obstante, até o momento não existe consenso a respeito da definição de apoio social, este conceito complexo, composto por várias dimensões que se associam com a saúde dos indivíduos.

Os termos mais freqüentemente encontrados na literatura são apoio social e redes sociais, mas é comum encontrarmos termos como relações sociais, integração social, vínculos sociais e ancoragem social, sendo empregados indistintamente 1,2. A despeito de uma grande quantidade de diferentes designações, a maioria dos investigadores concorda que tal categoria seria composta por uma estrutura e uma função.

As relações sociais formais e as informais são as duas dimensões que compõem a estrutura das relações sociais. A estrutura é formada pelos indivíduos com quem se tem uma relação interpessoal e pelas ligações entre esses indivíduos. As relações formais são relações mantidas devido à posição e papéis na sociedade. Incluem profissionais como médico, professor, advogado etc., e outras pessoas conhecidas. As relações sociais informais, também denominadas redes sociais, 
são compostas por todos os indivíduos (família, amigos, vizinhos, colegas de trabalho, comunidade etc.) e ligações entre indivíduos com quem se tem uma relação familiar próxima e/ou com envolvimento afetivo. A estrutura das relações sociais pode ser caracterizada sob diferentes aspectos tais como número e tipo de relações, freqüência de contatos, duração dos contatos, diversidade, densidade e reciprocidade.

Define-se a função das redes sociais como as interações interpessoais que ocorrem dentro da estrutura dessas redes. A função (apoio social propriamente) abrange aspectos qualitativos e comportamentais das relações sociais e compreende quatro tipos: (1) apoio emocional, que envolve expressões de amor e afeição; (2) apoio instrumental ou material que se refere aos auxílios concretos como provimento de necessidades materiais em geral, ajuda para trabalhos práticos (limpeza de casa, preparação de refeição, provimento de transporte) e ajuda financeira; (3) apoio de informação que compreende informações (aconselhamentos, sugestões, orientações) que podem ser usadas para lidar com problemas e resolvê-los; e (4) interação social positiva que diz respeito à disponibilidade de pessoas com quem se divertir e relaxar 1 .

Especialmente em idosos, numerosos estudos verificaram que o apoio social foi consistentemente associado com a mortalidade $3,4,5,6,7,8,9$, com algumas medidas de saúde, tais como capacidade funcional, incontinência urinária, autoavaliação de saúde e problemas visuais ${ }^{10}$, com sintomas depressivos 11,12, assim como com escores de auto-avaliação de satisfação com a vida 13,14, de estado de ânimo 10 e de auto-estima 15 .

Embora quase nenhuma atenção tenha sido dada ao apoio social como variável dependente, a compreensão das estruturas e dos processos sociais amplos, bem como dos processos psicológicos e biológicos, que determinam a quantidade e qualidade das relações sociais e de apoio na sociedade, pode ser considerada tão importante quanto o significado das relações sociais para a melhoria da saúde 16 .

Entre os poucos estudos que enfocam as redes sociais como variável desfecho observam-se algumas diferenças marcantes. Pessoas que trabalham em ocupações prestigiadas socialmente recebem maior número de apoio social 17; idosos com renda e nível educacional mais altos têm mais contatos com amigos, prestam apoio social com maior freqüência a outros e reportam maior satisfação com o apoio recebido dos membros da rede social de apoio 18; as redes sociais das mulheres são maiores e mais amplas do que a dos homens, que tendem a se relacionar exclusivamente com seus cônjuges 1 . Além disso, foram observadas diferenças significativas que favorecem os casados quanto ao apoio proveniente da família, tanto para os homens quanto para as mulheres 17 . Finalmente, foram encontradas por Due et al. ${ }^{1}$ diferenças relevantes na estrutura e na função das redes sociais segundo a idade: os contatos com seus pares são os mais freqüentes entre as pessoas de 25 anos, enquanto as de 70 anos têm mais contatos com filhos, amigos e pessoas conhecidas em associações formais.

Considerando-se a importância de se conhecer as estruturas e os processos sociais amplos que determinam a quantidade e qualidade das relações sociais e de apoio em população de idosos residentes em uma grande metrópole de um país em desenvolvimento, o presente estudo teve como objetivo investigar as redes sociais e a disponibilidade de apoio social instrumental informal, identificando a influência de algumas características sócio-econômicas e demográficas sobre elas.

\section{Métodos}

Este estudo é parte do inquérito sobre Saúde, Bem-estar e Envelhecimento (SABE) realizado na cidade de São Paulo, Brasil, em pessoas de 60 anos ou mais, durante o período de janeiro de 2000 a março de 2001. Trata-se de um estudo multicêntrico (em sete países da América Latina e Caribe) coordenado e financiado pela Organização Pan-Americana da Saúde (OPAS) e Organização Mundial da Saúde (OMS) e no Brasil, também com apoio financeiro da Fundação de Amparo à Pesquisa do Estado de São Paulo (FAPESP), executado por equipe da Faculdade de Saúde Pública da Universidade de São Paulo.

Foi utilizada amostragem probabilística em duas etapas. Na primeira, foram sorteados $72 \mathrm{se}$ tores censitários com probabilidade proporcional ao número de domicílios. Na segunda etapa, foram sorteados 90 domicílios por setor, tendo sido identificados 1.852 idosos. Desses, foram entrevistados 1.568, atingindo-se, após três visitas, a taxa de resposta esperada de $85 \% 19$.

Os dados da amostra foram ponderados para compensar diferenças nas probabilidades de seleção, originadas pela atualização das listas de endereços na segunda etapa do sorteio, e para ajustar a distribuição da amostra por sexo e idade aos dados populacionais obtidos no Censo Demográfico 200019.

Utilizou-se o programa Stata (Stata Corp., College Station, Estados Unidos) para as análises estatísticas e foram considerados os aspectos complexos do delineamento de amostragem, o sorteio de setores censitários e a ponderação. 
Quatro aspectos estruturais das redes sociais de apoio foram analisados como variáveis dependentes: (1) índice de freqüência de contatos (IFC); (2) índice de diversidade de contatos (IDC); (3) status de coabitação - variável dicotômica indicando se o idoso vivia só ou não; (4) situação conjugal - analisada como índice de rede social (casados; não-casados).

\section{Índice de freqüência de contatos (IFC)}

Corresponde ao número de contatos mensais e tem como objetivo avaliar o grau em que o idoso está conectado socialmente com outros. Este índice foi construído com base nas questões relacionadas com a freqüência de contatos com filhos, irmãos, outros familiares e com amigos, com um máximo de 301 contatos por mês e um mínimo de zero, dicotomizado em quartil inferior ( $<12$ contatos) e demais ( $\geq 12$ contatos).

\section{Índice de diversidade de contatos (IDC)}

Para avaliar a amplitude da rede social, foi construído a partir das mesmas questões do índice anterior, considerando-se, entretanto, contato regular, ocorrido pelo menos duas vezes por mês, de determinado tipo (contatos com filho, com irmão, com pais, com sogros, com genro/nora, com neto, com sobrinho, com outro familiar e com amigos), com um máximo de quatro tipos de contatos e um mínimo de zero. Se nenhuma das questões sobre a freqüência de contatos foi respondida, os índices foram considerados "não resposta". Por outro lado, se as respostas eram disponíveis para pelo menos um tipo de contato, a "não resposta", individualmente, foi tratada como se não tivesse havido contato e conseqüentemente codificada como zero. Os índices foram expressos em quartis e depois dicotomizados: quartil inferior $(<1$ contato regular) e demais ( $\geq 1$ contato regular). Os índices de contato e de diversidade foram construídos segundo metodologia de Lund et al. ${ }^{4}$.

Além disso, os índices de freqüência de ajudas recebidas (IFAR) e prestadas (IFAP), dimensões funcionais da rede social de apoio, foram estudadas também como variáveis dependentes. Apoio efetivamente recebido (máximo de 280 e mínimo de zero) e apoio efetivamente prestado (máximo de 336 e mínimo de zero), tendo as duas variáveis o mesmo ponto de corte do quartil inferior, foram dicotomizadas em quartil inferior ( $<28$ ajudas) e demais ( $\geq 28$ ajudas).

Entre as variáveis independentes foram estudadas as seguintes características sócio-demográficas: idade em anos (faixas de 60 a 64, 65 a 69, 70 a 74,75 a 79 e 80 anos ou mais); renda mensal do idoso per capita em Reais - soma dos rendimentos declarados pelo entrevistado dividido pelo número de pessoas que dependia desta renda - (ordenada de forma crescente e estratificada em quartis, 1 o: $<\mathrm{R} \$ 75,50 ; 2$ o: $\mathrm{R} \$ 75,50 \leq$ renda per capita $<\mathrm{R} \$ 151,00$ e 3ㅇ/4o: $\geq \mathrm{R} \$ 151,00$ ); e escolaridade em número de anos de estudo (nunca foi à escola, 1 a 3 anos, 4 anos e 5 anos ou mais). O estado conjugal foi tratado, primeiro, como índice de apoio social e, segundo, como variável independente, característica demográfica, de modo a estimar as diferenças dos outros aspectos do apoio social segundo as particularidades relacionadas ao casamento. Como variável demográfica, o estado conjugal foi categorizado em casado, solteiro, separado/divorciado e viúvo.

Com base naquelas pesquisas que têm fornecido fortes evidências para uma importante diferença quanto ao gênero, optou-se para a análise dos resultados separando-se os homens das mulheres.

Para a identificação da associação individualizada entre cada aspecto das redes sociais e as variáveis demográficas e sócio-econômicas foi realizada análise de regressão logística múltipla 20. $\mathrm{Na}$ interpretação dos resultados, considerou-se o nível de significância de 0,05.

\section{Resultados}

Nas Tabelas 1, 2 e 3 estão apresentados os resultados obtidos na análise de cada uma das variáveis de rede social para mulheres e homens separadamente. Note-se que foram apresentadas nas tabelas somente as variáveis independentes que evidenciaram associação na análise bivariada, cujos resultados não foram aqui representados.

\section{Índice de freqüência de contatos (IFC) e índice de diversidade de contatos (IDC)}

As mulheres de 80 anos ou mais, comparadas com as mais jovens que 65 anos, apresentaram significativamente maiores chances de ter baixas freqüências de contatos, enquanto para os homens a idade não mostrou exercer influência alguma sobre o número de contatos. Quanto ao estado conjugal, relativamente aos casados, mulheres e homens solteiros mostraram chances bastante elevadas e significativas de terem baixas freqüências de contatos. Para as mulheres, os fatores renda per capita e escolaridade não mostraram efeitos sobre o IFC. Constatou-se que os homens de renda mais baixa têm chance significativamente aumentada para apresentarem menores freqüências de contatos, comparativamente aos de rendas do $3 \underline{\text { o e }}$ 4o quartis. 
Associação entre o índice de freqüência de contatos (IFC) e índice de diversidade de contatos (IDC) dos idosos e os fatores demográficos e sócio-econômicos - modelo de regressão logística múltipla. Município de São Paulo, Brasil, 2000-2001.

\begin{tabular}{|c|c|c|c|c|}
\hline \multirow[t]{3}{*}{ Variáveis (\%) } & \multirow{2}{*}{\multicolumn{2}{|c|}{$\begin{array}{l}\text { IFC (baixa freqüência) } \\
\text { Odds ajustado (IC95\%) }\end{array}$}} & \multirow{2}{*}{\multicolumn{2}{|c|}{$\begin{array}{l}\text { IDC (pequena diversidade) } \\
\text { Odds ajustado (IC95\%) }\end{array}$}} \\
\hline & & & & \\
\hline & Mulheres & Homens & Mulheres & Homens \\
\hline Idade (anos) & & * & & * \\
\hline $60-64$ & 1,00 & & 1,00 & \\
\hline $65-69$ & $0,89(0,50-1,62)$ & & $1,82(0,85-3,92)$ & \\
\hline $70-74$ & $1,06(0,62-1,81)$ & & $1,81(0,80-4,11)$ & \\
\hline $75-79$ & $1,08(0,59-1,97)$ & & $2,22(1,05-4,69)$ & \\
\hline 80 ou mais & $2,41(1,36-4,29)$ & & $4,67(2,51-8,70)$ & \\
\hline \multicolumn{5}{|l|}{ Estado conjugal } \\
\hline Casado & 1,00 & 1,00 & 1,00 & 1,00 \\
\hline Solteiro & $5,73(2,43-13,50)$ & $8,93(3,28-24,34)$ & $6,60(2,83-15,40)$ & $8,89(3,11-25,41)$ \\
\hline Separado/Divorciado & $1,77(0,94-3,31)$ & $2,26(0,98-5,24)$ & $1,71(0,77-3,79)$ & $1,50(0,51-4,45)$ \\
\hline Viúvo & $1,34(0,86-2,10)$ & $1,73(0,84-3,54)$ & $0,71(0,45-1,13)$ & $1,02(0,34-3,05)$ \\
\hline Renda per capita mensal (Reais) & ** & & ** & \\
\hline 3ㅇ e 4 으 quartil & & 1,00 & & 1,00 \\
\hline 2o quartil & & $1,32(0,81-2,13)$ & & $1,31(0,63-2,72)$ \\
\hline 1o quartil & & $2,54(1,36-4,73)$ & & $4,97(2,54-9,69)$ \\
\hline Escolaridade (anos de estudo) & $\star \star$ & * & $\star \star$ & * \\
\hline \multicolumn{5}{|l|}{5 ou mais } \\
\hline \multicolumn{5}{|l|}{4} \\
\hline \multicolumn{5}{|l|}{$1-3$} \\
\hline Nunca foi à escola & & & & \\
\hline
\end{tabular}

* As variáveis faixa etária e escolaridade foram retiradas do modelo ajustado dos homens por não se associarem significativamente com os índices em questão, em análise univariada;

** As variáveis renda e escolaridade foram retiradas dos modelos ajustados das mulheres por não se associarem significativamente com os índices em questão, em análise univariada.

Os resultados em relação ao IDC foram muito semelhantes aos do IFC, destacando-se aí apenas o fato de que as mulheres mostraram chance significativamente maior de apresentarem pequena diversidade de contatos a partir dos 75 anos de idade.

\section{Status de coabitação}

Observou-se alta colinearidade entre as variáveis "status de coabitação" e "estado conjugal" e por esta razão, esta variável não foi mantida na regressão múltipla.

A idade mostrou efeito significativo sobre o status de coabitação, para ambos os gêneros, a partir do grupo etário de 70 a 74 anos de idade, comparando-se com o grupo de 60 a 64 anos. É interessante notar que as chances de "morar só", entre as mulheres, são semelhantes em todas as faixas etárias, entretanto para os homens a chance para a mesma condição é maior que entre as mulheres e aumenta gradativamente de 3,55 para 4,5 vezes maior, conforme aumenta a faixa etária.

Por outro lado, para ambos os gêneros, a chance de "morar só" foi significativamente menor em rendas mais baixas, comparadas com rendas do $3 \circ$ e 4 quartis. Chama a atenção que as mulheres do patamar mais baixo de renda tenham apresentado a menor chance (muito próxima de zero) de estarem na condição "não-casadas", quando comparadas intra e inter-gêneros.

Tanto entre as mulheres quanto entre os homens, a escolaridade não mostrou influência significativa no status de coabitação, não tendo sido inserida no modelo ajustado.

\section{Estado conjugal (não-casado)}

A chance de se encontrar o idoso na condição "não casado" é significativamente maior no grupo etário de 80 anos ou mais, comparando-se 
Associação entre status de coabitação/estado conjugal dos idosos e os fatores demográficos e sócio-econômicos - modelo de regressão logística múltipla. Município de São Paulo, Brasil, 2000-2001.

\begin{tabular}{|c|c|c|c|c|}
\hline \multirow[t]{3}{*}{ Variáveis (\%) } & \multirow{2}{*}{\multicolumn{2}{|c|}{$\begin{array}{l}\text { Status de coabitação (moram só) } \\
\text { Odds ajustado (IC95\%) }\end{array}$}} & \multirow{2}{*}{\multicolumn{2}{|c|}{$\begin{array}{c}\text { Estado conjugal (não casados) } \\
\text { Odds ajustado (IC95\%) }\end{array}$}} \\
\hline & & & & \\
\hline & Mulheres & Homens & Mulheres & Homens \\
\hline \multicolumn{5}{|l|}{ Idade (anos) } \\
\hline $60-64$ & 1,00 & 1,00 & 1,00 & 1,00 \\
\hline $65-69$ & $1,51(0,81-2,81)$ & $2,06(0,58-7,35)$ & $1,18(0,73-1,92)$ & $1,11(0,65-1,90)$ \\
\hline $70-74$ & $2,05(1,15-3,66)$ & $3,55(1,09-11,58)$ & $1,68(0,99-2,85)$ & $0,99(0,59-1,65)$ \\
\hline $75-79$ & $2,72(1,50-4,94)$ & $3,85(1,18-12,51)$ & $1,83(1,00-3,34)$ & $1,49(0,74-2,99)$ \\
\hline 80 ou mais & $2,58(1,33-4,99)$ & $4,50(1,41-14,33)$ & $4,83(2,45-9,52)$ & $3,12(1,88-5,18)$ \\
\hline Renda per capita mensal (Reais) & & & & * \\
\hline 3o e 4o quartil & 1,00 & 1,00 & 1,00 & \\
\hline 2o quartil & $0,18(0,11-0,31)$ & $0,30(0,11-0,82)$ & $0,38(0,24-0,60)$ & \\
\hline 1ㅇ quartil & $0,03(0,01-0,09)$ & $0,19(0,02-1,51)$ & $0,11(0,07-0,17)$ & \\
\hline Escolaridade (anos de estudo) & $\star \star$ & $\star \star$ & & * \\
\hline 5 ou mais & & & 1,00 & \\
\hline 4 & & & $1,74(0,98-3,09)$ & \\
\hline $1-3$ & & & $2,11(1,12-3,99)$ & \\
\hline Nunca foi à escola & & & $2,96(1,58-5,52)$ & \\
\hline
\end{tabular}

* As variáveis renda e escolaridade não se associaram significativamente com a variável estado conjugal, e, portanto, foram retiradas do modelo ajustado;

** A variável escolaridade não se associou significativamente com o status de coabitação e, portanto, foi retirada do modelo ajustado.

com o de 60 a 64 anos, sendo que para as mulheres, nessa faixa etária, a chance de estarem nessa condição é 1,5 vez maior que entre os homens do mesmo grupo etário.

Os níveis de renda e o grau de escolaridade mostraram associação significativa com o "estado conjugal" somente entre as mulheres.

Comparando-se com as mulheres classificadas nas rendas mais altas (3o e $4 \underline{\text { o quartis), todas }}$ as de níveis inferiores de renda apresentaram chances significativamente menores de estarem na condição "não-casadas".

Entre as mulheres, a chance de ser "não-casada" dobra e triplica entre as que tinham de 1 a 3 anos de escolaridade e naquelas que nunca freqüentaram a escola, respectivamente, comparadas àquelas com grau de escolaridade maior ou igual a cinco anos.

\section{Índice de freqüência de ajudas recebidas (IFAR)}

A idade mostrou-se associada ao IFAR somente entre as mulheres, sendo que em comparação àquelas do grupo etário mais jovem, todas as outras apresentaram menores chances de terem baixas freqüências de ajudas recebidas, embora com significância estatística apenas na faixa de 80 anos ou mais.
Em relação ao estado conjugal, para ambos os gêneros, as chances de apresentarem baixa freqüência de ajudas recebidas mostraram-se consistentemente aumentadas para "solteiro", "separado/divorciado" e "viúvo", quando comparadas à categoria "casado”. Chamam a atenção os valores triplicados dos odds referentes aos homens relativamente às mulheres.

Entre as mulheres classificadas nos níveis de renda inferiores as chances de apresentarem baixa freqüência de ajudas recebidas foram significativamente menores em comparação com as de rendas do 3o e 4o quartis. Para os homens, a renda não mostrou influência significativa sobre o IFAR.

A escolaridade, que na análise bivariada mantinha relação estatisticamente significativa entre as mulheres, no modelo ajustado perdeu a significância, ou seja, esta variável não mostrou associação com o IFAR, independentemente da idade, do estado conjugal e da renda. Para os homens, o grau de escolaridade não se mostrou associado com o IFAR.

\section{Índice de freqüência de ajudas prestadas (IFAP)}

Constatou-se que a idade influencia o IFAP, sendo que para as mulheres, a partir da faixa etária 
Associação entre índice de ajudas recebidas (IFAR)/índice de ajudas prestadas (IFAP) dos idosos e os fatores demográficos e sócio-econômicos - modelo de regressão logística múltipla. Município de São Paulo, Brasil, 2000-2001.

\begin{tabular}{|c|c|c|c|c|}
\hline \multirow[t]{3}{*}{ Variáveis (\%) } & \multirow{2}{*}{\multicolumn{2}{|c|}{$\begin{array}{l}\text { IFAR (baixa freqüência) } \\
\text { Odds ajustado (IC95\%) }\end{array}$}} & \multirow{2}{*}{\multicolumn{2}{|c|}{$\begin{array}{l}\text { IFAP (baixa freqüência) } \\
\text { Odds ajustado (IC95\%) }\end{array}$}} \\
\hline & & & & \\
\hline & Mulheres & Homens & Mulheres & Homens \\
\hline Idade (anos) & & * & & \\
\hline $60-64$ & 1,00 & & 1,00 & 1,00 \\
\hline $65-69$ & $0,83(0,48-1,41)$ & & $0,93(0,48-1,78)$ & $0,87(0,44-1,73)$ \\
\hline $70-74$ & $0,80(0,50-1,30)$ & & $1,95(1,03-3,71)$ & $1,14(0,68-1,88)$ \\
\hline $75-79$ & $0,99(0,60-1,62)$ & & $2,26(1,15-4,44)$ & $1,02(0,55-1,89)$ \\
\hline 80 ou mais & $0,30(0,14-0,67)$ & & $4,45(2,26-8,77)$ & $3,22(1,66-6,28)$ \\
\hline \multicolumn{5}{|l|}{ Estado conjugal } \\
\hline Casado & 1,00 & 1,00 & 1,00 & 1,00 \\
\hline Solteiro & $2,10(0,88-4,97)$ & $6,11(2,17-17,17)$ & $5,79(2,45-13,69)$ & $6,73(2,63-17,23)$ \\
\hline Separado/Divorciado & $1,76(1,03-3,00)$ & $3,26(1,49-7,11)$ & $3,00(1,20-7,50)$ & $3,87(2,02-7,38)$ \\
\hline Viúvo & $1,66(1,05-2,61)$ & $4,50(1,99-10,19)$ & $3,54(1,87-6,72)$ & $3,25(1,72-6,13)$ \\
\hline Renda per capita mensal (Reais) & & * & & * \\
\hline 3으 e 4 ㅇ quartil & 1,00 & & 1,00 & \\
\hline 2o quartil & $0,44(0,26-0,72)$ & & $0,38(0,24-0,60)$ & \\
\hline 1ㅇ quartil & $0,33(0,17-0,62)$ & & $0,28(0,14-0,55)$ & \\
\hline Escolaridade (anos de estudo) & & * & * & * \\
\hline 5 ou mais & 1,00 & & & \\
\hline 4 & $0,89(0,49-1,62)$ & & & \\
\hline $1-3$ & $0,69(0,34-1,39)$ & & & \\
\hline Nunca foi à escola & $0,59(0,28-1,22)$ & & & \\
\hline
\end{tabular}

* Variáveis retiradas do modelo ajustado por não apresentarem associação significativa com as variáveis desfechos nas análises univariadas.

de 65 a 69 anos, as chances de elas apresentarem baixas freqüências de ajudas prestadas aumentaram significativamente, comparando-se com a faixa de 60 a 64 anos. Para os homens, somente no grupo etário mais avançado tal chance foi significativamente elevada.

Para ambos os gêneros, o estado civil apresentou forte associação com este tipo de apoio funcional. "Solteiros", "separados/divorciados" e "viúvos" têm chances significativamente maiores de apresentarem baixa freqüência de ajudas prestadas.

Entre as mulheres, a renda afeta o IFAP; sendo que aquelas de níveis de rendimento mais baixos apresentaram chances significativamente menores de haver baixas freqüências, tomandose como referência rendas do $3^{\circ}$ e $4^{\circ}$ quartis. Para os homens, a renda não mostrou associação com este índice.

Para ambos os gêneros, não houve associação entre escolaridade e IFAP.

\section{Apoio funcional e rede social (estrutural)}

Para testar o efeito dos aspectos estruturais das redes sobre o apoio funcional incluíram-se, na análise de regressão múltipla, as variáveis relativas à estrutura (IFC, IDC e status de coabitação) e as sócio-demográficas (variáveis de controle), que se associaram na análise bivariada com o apoio social funcional (IFAR e IFAP). Esses resultados estão apresentados na Tabela 4.

Para ambos os gêneros, o IFC e o IDC não mostraram associação com o IFAR, não foram, portanto, incluídos na regressão múltipla.

Em relação ao status de coabitação, mulheres e homens que moravam sozinhos mostraram significativamente maiores chances de apresentar baixas freqüências de ajudas recebidas. Entre os homens, essas mesmas chances são encontradas nas condições do estado conjugal "solteiro" e "viúvo". Ao contrário, entre as mulheres, o fator estado conjugal que mostrou forte associação independente com o IFAR (Tabela 3), perdeu sua significância quando ajustado com a variável status de coabitação. 
Associação entre apoio funcional e apoio estrutural - modelo de regressão logística múltipla. Município de São Paulo, Brasil, $2000-2001$.

\begin{tabular}{|c|c|c|c|c|}
\hline \multirow[t]{3}{*}{ Variáveis (\%) } & \multicolumn{2}{|c|}{ IFAR (baixa freqüência) } & \multicolumn{2}{|c|}{ IFAP (baixa freqüência) } \\
\hline & Odds ajustado (IC95\%) * & Odds ajustado (IC95\%) ** & Odds ajustado (IC95\%) *** & Odds ajustado (IC95\%) \# \\
\hline & Mulheres & Homens & Mulheres & Homens \\
\hline ÍFC & $\# \#$ & $\# \#$ & & \\
\hline Alta freqüência & & & 1,00 & 1,00 \\
\hline Baixa freqüência & & & $1,86(1,12-3,07)$ & $1,09(0,67-1,77)$ \\
\hline ÍDC & \#\# & \#\# & \#\# & \\
\hline Grande diversidade & & & & 1,00 \\
\hline Pequena diversidade & & & & $1,87(0,93-3,78)$ \\
\hline \multicolumn{5}{|l|}{ Status de coabitação } \\
\hline Não mora só & 1,00 & 1,00 & 1,00 & 1,00 \\
\hline Mora só & $6,66(4,00-11,08)$ & $4,60(2,09-10,13)$ & $10,43(6,32-17,21)$ & $8,37(3,18-21,99)$ \\
\hline \multicolumn{5}{|l|}{ Estado conjugal } \\
\hline Casado & 1,00 & 1,00 & 1,00 & 1,00 \\
\hline Solteiro & $1,05(0,46-2,40)$ & $3,33(1,20-9,19)$ & $2,42(0,88-6,66)$ & $2,36(0,77-7,26)$ \\
\hline Separado/Divorciado & $1,07(0,59-1,94)$ & $1,83(0,80-4,22)$ & $1,60(0,56-4,63)$ & $1,80(0,89-3,63)$ \\
\hline Viúvo & $0,96(0,59-1,55)$ & $2,73(1,11-6,74)$ & $1,82(0,91-3,62)$ & $1,75(0,82-3,73)$ \\
\hline
\end{tabular}

IFAR: índice de freqüência de ajudas recebidas; IFAP: índice de freqüência de ajudas prestadas; IFC: índice de freqüência de contatos; IDC: índice de diversidade de contatos.

* Ajustado por idade, renda, status de coabitação e estado conjugal;

** Ajustado por idade, status de coabitação e estado conjugal;

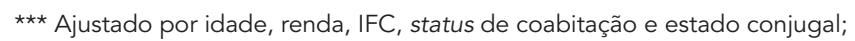

\# Ajustado por idade, IFC, IDC, status de coabitação e estado conjugal;

\#\# Variáveis retiradas do modelo ajustado por não se associarem significativamente com a variável ajudas recebidas, na análise univariada.

No que se refere ao IFAP, a chance, no caso das mulheres, de elas apresentarem baixa freqüência de ajudas prestadas foi significativamente maior entre as que tinham baixas freqüências de contatos. Para os homens, o IFC e o IDC mostraram associação significativa com o IFAP na análise bivariada, entretanto, isto não se manteve quando controlado por outras variáveis.

Chama a atenção, entre os resultados da Tabela 4, o efeito do status de coabitação sobre o IFAP, que evidencia chances muito altas, para homens e mulheres que "moram sós", de apresentarem freqüências baixas de ajudas prestadas.

A forte associação do fator estado conjugal com o IFAP (Tabela 3) se perdeu quando estatisticamente controlado para os efeitos da variável "status de coabitação".

Entre os homens, os fatores IFC, IDC e estado conjugal mostraram-se associados com o IFAP, entretanto não mantiveram a mesma magnitude de associação quando ajustados entre si.

\section{Discussão}

A relevância do presente estudo consiste no fato de que se tem pouco conhecimento sobre a distribuição das redes sociais na população de idosos e sobre a maneira como as características demográficas e sociais influenciam nesta distribuição. Este é um dos poucos estudos em que a relação entre fatores sócio-demográficos e um abrangente conjunto de medidas das redes sociais de apoio foi avaliado, com referência nos dados de uma pesquisa de base populacional do Município de São Paulo.

Procurou-se discutir os resultados enfatizando diferenças e similaridades com a literatura, entretanto esta tarefa foi dificultada pela exigüidade de investigações deste tipo e pela característica do apoio social, um constructo multifacetado, que contém diferentes dimensões, o que torna difícil a comparabilidade dos resultados. Nesse sentido, a mensuração das redes sociais de apoio pode ser apontada como uma fragilidade deste estudo. Embora a mensuração aqui empregada venha sendo amplamente utilizada, há que se reconhecer que diversas questões metodoló- 
gicas relativas à operacionalização do conceito de redes sociais de apoio ainda não estão completamente resolvidas. Ademais, como o projeto SABE visava investigar fundamentalmente os arranjos domiciliares e o intercâmbio de apoio entre o idoso e a sua família, a construção do instrumento de mensuração visando abarcar outros aspectos das redes sociais de apoio foi totalmente secundária à coleta dos dados. Alguns problemas advindos dessa situação foram detectados e devidamente analisados ao longo desta discussão.

Diversos estudos confirmam a existência de diferenças na estrutura das relações sociais conforme a idade 1,21. Em termos gerais, pessoas mais jovens tendem a ter mais contatos e mais apoio instrumental do que os mais idosos. Esses achados foram consistentes com os desta investigação somente no caso das mulheres, para as quais a freqüência de diversidade dos contatos foi menor nas mais velhas (80 anos ou mais). Nos grupos etários mais avançados, o nível dos contatos piora, provavelmente pela diminuição do número de irmãos e amigos vivos, que constituíam sua rede social potencial 21 .

$\mathrm{O}$ aumento da idade também piorou os outros aspectos da rede social de apoio. Ou seja, as chances dos idosos de "morarem sós", de serem "não casados" e de apresentarem baixa freqüência no IFAP aumentaram significativamente, sobretudo na faixa acima de 70 anos. A morte de um dos cônjuges é a principal razão que pode levar o idoso a morar sozinho, o que é mais provável de ocorrer em idades mais avançadas, e o que, por conseguinte, pode explicar a maior proporção das condições "não casado" e "morar só” entre os mais velhos. O declínio no número de contatos pessoais e a situação de "morarem sós" necessitam de uma análise mais aprofundada para que se possa compreendê-los no contexto dos idosos. A tendência de os idosos morarem sozinhos pode significar um novo tipo de arranjo 22, no qual, ainda, a troca e a assistência ocorrem de maneira intensa (o que alguns autores denominam "intimidade à distância"), facilitadas pelo aperfeiçoamento das formas de comunicação à distância 23 .

A elevação observada nos níveis de ajudas recebidas conforme o aumento da idade, para ambos os gêneros, parece estar relacionada com a necessidade do idoso de receber algum tipo de ajuda, porque nessa faixa etária é esperado que o idoso passe a ser menos independente funcionalmente. Esses achados são consistentes com a observação de alguns estudos de coorte onde o aparecimento de incapacidades funcionais no idoso contribui para a formação de uma rede de apoio 24 . Em relação à diminuição nos níveis de ajudas prestadas, pode ser inferido que a inca- pacidade funcional, em conseqüência da idade, impede o fornecimento de ajudas.

Homens e mulheres na condição conjugal "solteiro" experienciam piores níveis de apoio social no que diz respeito à freqüência de contatos, à diversidade desses contatos e ao número de ajudas recebidas e prestadas.

A explicação para esses níveis encontrados nos solteiros poderia estar relacionada à maneira como foi construído o questionário onde se privilegiaram as trocas sociais (recebe ou dá ajuda em dinheiro, em comida, em roupa ou em serviços) e não os contatos sociais em si (visitas sociais, amigos com quem faz passeios ou alguma outra atividade de lazer) e portanto, os contatos que não envolviam apoio instrumental não foram considerados. Entretanto, o que estes resultados parecem denotar é que os filhos são pessoaschave no apoio a estes idosos e que, portanto a presença ou ausência de filhos na rede de apoio tem um forte impacto sobre o apoio estrutural e funcional desta população, o que deixa o idoso que não tem filho (provavelmente o solteiro) em desvantagem.

Estudos anteriores observaram que, entre os idosos, melhores níveis de apoio associam-se com melhores níveis de rendas, independentemente do gênero 17. Entretanto, os resultados do presente estudo concordaram com esses autores somente em relação aos homens que, quando classificados nos níveis de rendas inferiores, tiveram significativamente piores níveis de contatos e de diversidade nesses contatos.

Embora homens idosos que tinham rendas nos patamares mais baixos tenham apresentado piores níveis em diversos aspectos da rede social de apoio, pode-se dizer que renda mais baixa é fator que "protege" o idoso, notoriamente, em relação à coabitação e ao apoio funcional. A explicação para esses resultados poderia estar relacionada com os motivos que levam à conformação nos arranjos familiares dos idosos. Situações como incapacidade física ou financeira; dependência econômica dos filhos para constituição de novo domicílio; necessidade de um responsável pelos netos e pela própria casa, em virtude do trabalho externo dos filhos 22 são apontadas como fatores que levam mais da metade dos idosos do Município de São Paulo, em geral de baixo nível sócio-econômico, a residir com filhos 25,26. Portanto, as famílias plurigeracionais cumpririam as funções de reunir renda para satisfazer melhor as possibilidades de utilização de bens e serviços e de facilitar, pela proximidade física, as transferências de apoio.

A verificação do efeito da estrutura das redes sociais sobre o apoio funcional teve como hipótese subjacente que aspectos estruturais das 
redes sociais são especialmente relevantes para populações idosas porque não só proporcionam oportunidades de contatos, mas também fornecem o contexto no qual os apoios instrumental e emocional são recebidos.

A expectativa da influência do IFC e do IDC sobre o apoio funcional não foi confirmada totalmente. Os resultados do presente estudo indicaram que somente entre as mulheres a baixa freqüência de contatos mostrou associação com baixa freqüência de ajudas prestadas. Achados anteriores mostraram que o número de contatos afeta significativamente diversos tipos de apoio funcional, inclusive ajudas recebidas 27 . A explicação para a discordância dos resultados deste estudo pode estar relacionada com o fato de que o índice de ajudas recebidas/prestadas considerou as ajudas recebidas/prestadas pelos co-residentes e, por outro lado, para o cálculo do índice de contatos foram levados em consideração contatos com pessoas residentes fora do domicílio. Como as transferências intrafamiliares são intensas, neste caso, o resultado pode ser espúrio.

O status de coabitação apresentou uma intensa influência sobre o índice de ajudas recebidas em ambos os gêneros. Os idosos que moram sozinhos têm muito maior chance de apresentar níveis mais baixos de ajudas recebidas do que aqueles que moram com outra pessoa.

Aqui, vale assinalar uma questão teórica e metodológica no que diz respeito ao instrumento empregado para medir o apoio instrumental. É possível que se esse tipo de apoio tivesse sido medido por meio de um instrumento que avalia o apoio percebido e não o auxílio "concreto", os resultados poderiam ser diferentes. É possível que os idosos que residem sozinhos reúnam diversas condições financeiras e de saúde favoráveis que façam com que não tenham necessidades materiais em geral, ajuda para trabalhos práticos ou ajuda financeira. Portanto, esta medida de ajudas recebidas (IFAR) pode não ser o mais adequado para avaliar o nível do apoio funcional nestes idosos.
É interessante notar que as chances de ocorrência de baixa freqüência de ajudas recebidas são significativamente maiores entre os "não casados", entretanto, quando houve ajustamento de "estado conjugal" pelo "status de coabitação”, a associação só se manteve para os homens. Isto significa que, para as mulheres, o "status de coabitação" teve maior peso em relação ao "estado civil” e para os homens, as chances de não receber ajudas são maiores entre os solteiros e viúvos, independentemente da situação "morar só”.

Embora tenha que se notar a necessidade de outros estudos que incluam dimensões qualitativas das redes sociais para melhor compreendê-las, um achado deste estudo que vale ser ressaltado, entre outros, é a condição dos idosos que foram classificados em níveis de rendas mais baixos. Estes apresentaram, por um lado, maiores chances de apresentarem piores níveis de integração social (poucos contatos não diversificados) e por outro lado, menores chances de estarem na condição "morar só” e de apresentarem baixas freqüências de apoio funcional. É possível que a situação desse idoso seja daquele que está mais incapacitado funcionalmente e, portanto, não pode estar residindo em domicílio unipessoal e depende do apoio informal prestado pela família. Ações com vistas à prevenção e promoção de saúde, voltadas especificamente aos idosos e seus familiares, poderiam ser planejadas com o apoio das unidades básicas de saúde sob a estratégia Saúde da Família, elegendo o domicílio plurigeracional como um marcador capaz de identificar idosos com alto risco de perdas funcionais graves.

Os resultados deste estudo sugerem também que os conhecimentos sobre as redes sociais em idosos poderiam ser melhorados por estudos que incluam dimensões qualitativas das redes sociais, como avaliações subjetivas do apoio, e aspectos negativos como conflitos e tensões, componentes aqui não abrangidos. 


\section{Resumo}

Este trabalho teve como objetivo descrever a distribuição das redes sociais e de apoio em idosos no Município de São Paulo, Brasil, por características sócio-econômicas e demográficas. Estudou-se amostra probabilística de 1.568 idosos (60 ou mais anos) da cidade de São Paulo (inquérito sobre Saúde, Bem-estar e Envelhecimento - SABE), focalizando-se as dimensões estruturais e funcionais das redes sociais. Para a análise dos dados utilizou-se a regressão logística simples e múltipla. As melhores condições nas redes sociais foram observadas nos menores patamares de renda e as piores entre os mais velhos e os "não casados". Para as mulheres, as chances de estarem "não casadas" aumentaram significativamente quanto pior era o seu nível educacional. O "status de coabitação" foi o que mais influenciou o apoio funcional, onde "morar só" aumentou significativamente as chances de não ocorrerem as trocas sociais. Os modelos de regressão logística múltipla construídos separadamente por gênero sugerem que idade, estado civil, renda per capita e escolaridade estão associados com as redes sociais entre os idosos. No entanto, essas relações manifestam-se de modos desiguais entre gêneros e entre as características sócio-demográficas para as diferentes dimensões da rede social.

Apoio Social; Idoso; Condições Sociais

\section{Colaboradores}

T. E. C. Rosa idealizou o trabalho, realizou a análise de dados, elaborou o roteiro do artigo e redigiu o texto final. M. H. D’A. Benício colaborou na análise de dados e na redação do texto final. M. C. G. P. Alves realizou a ponderação da amostra, colaborou na análise de dados e na redação do texto final do texto. M. L. Lebrão coordenou o estudo multicêntrico SABE (Saúde, Bem-estare Envelhecimento) que gerou estes dados.

\section{Referências}

1. Due P, Holstein B, Lund R, Modvig J, Avlund K. Social relations: network, support and relational strain. Soc Sci Med 1999; 48:661-73.

2. Castro R, Campero L, Hernández B. La investigación sobre apoyo social en salud: situación actual y nuevos desafíos. Rev Saúde Pública 1997; 31:42535.

3. Ceria CD, Masaki KH, Rodriguez BL, Chen R, Yano $\mathrm{K}$, Curb JD. The relationship of psychosocial factors to total mortality among older Japanese-American men: the Honolulu Heart Program. Am J Geriatr Soc 2001; 49:725-31.

4. Lund R, Modvig J, Due P, Holstein BE. Stability and change in structural social relations as preditor of mortality among elderly women and men. Eur J Epidemiol 2000; 16:1087-97.

5. Rahman MO. Age and gender variation in the impact of household structure on elderly mortality. Int J Epidemiol 1999; 28:485-91.
6. Davis MA, Moritz DJ, Neuhaus JM, Barclay JD, Gee L. Living arrangements, changes in living arrangements, and survival among community dwelling older aldults. Am J Public Health 1997; 87:371-7.

7. Yasuda N, Zimmerman SI, Hawkes W, Fredman L, Hebel JR, Magaziner J. Relation of social network characteristics to 5-year mortality among youngold versus old-old white women in an urban community. Am J Epidemiol 1997; 145:516-23.

8. Penninx BW, van Tilburg T, Kriegsman DM, Deeg DJ, Boeke AJ, van Eijk JT. Effects of social support and personal coping resources on mortality in older age: the Longitudinal Aging Study Amsterdam. Am J Epidemiol 1997; 146:510-9.

9. Shye D, Mullooly JP, Freeborn DK, Pope CR. Gender differences in the relationship between social network support and mortality: a longitudinal study of an elderly cohort. Soc Sci Med 1995; 41:935-47. 
10. Litwin H. Social network type and morale in old age. Gerontologist 2001; 41:516-24.

11. Wallsten SM, Tweed DL, George LK. Disability and depressive symptoms in the elderly: the effects of instrumental support and its subjective appraisal. Int J Aging Hum Dev 1999; 48:145-59.

12. Palinkas LA, Wingard DL, Barret-Connor E. The biocultural context of social networks and depression among the elderly. Soc Sci Med 1990; 30:4417.

13. Pinquart M, Sörensen S. Influences of socioeconomic status, social network, and competence on subjective well-being in later life: a meta-analysis. Psychol Aging 2000; 15:187-224.

14. McMamish-Svensson C, Samuelsson G, Hagberg B, Svensson T, Dehlin O. Social relationships and health as predictors of life satisfaction in advanced old age: results from a Swedish longitudinal study. Int J Aging Hum Dev 1999; 48:301-24

15. Lee GR, Shehan CL. Social relations and the selfesteem of older persons. Res Aging 1989; 11:42742.

16. House SJ, Landis KR, Umberson D. Social relationships and health. Science 1988; 241:540-5.

17. Turner RJ, Marino F. Social support and social structure: a descriptive epidemiology. J Health Soc Behav 1994, 35:193-212.

18. Krause N. Borawski-Clark E. Social class differences in social support among older adults. Gerontologist 1995; 35:498-508

19. Silva NN. Aspectos metodológicos: processo de amostragem. In: Lebrão ML, Duarte YAO, organizadores. SABE - Saúde, Bem-estar e Envelhecimento - o projeto SABE no município de São Paulo: uma abordagem inicial. Brasília: Organização Pan-Americana da Saúde; 2003. p. 47-57.
20. Hosmer DM, Lemeshow S. Applied logistic regression. New York: John Wiley and Sons; 1989.

21. Olsen O, Iversen L, Sabroe S. Age and the operationalization of social support. Soc Sci Med 1991; 32:767-71

22. Yazaki LM, Melo AV, Ramos LR. Perspectivas atuais do papel da família frente ao envelhecimento populacional: um estudo de caso. In: Fundação Sistema Estadual de Análise de Dados, organizador. A população idosa e o apoio familiar. São Paulo: Fundação Sistema Estadual de Análise de Dados; 1991. p. 11-86. (Informe Demográfico, 24).

23. Debert GG. A construção e a reconstrução da velhice: família, classe social e etnicidade. In: Neri AL, Debert GG, organizadores. Velhice e sociedade. Campinas: Editora Papirus; 1999. p. 41-68.

24. Litwin H. Social network type and health status in a national sample of elderly Israelis. Soc Sci Med 1998; 46:599-609.

25. Rosa TEC, Benício MHD'A, Latorre MRDO, Ramos LR. Fatores determinantes da capacidade funcional entre idosos. Rev Saúde Pública 2003; 37:40-8.

26. Saad PM. Arranjos domiciliares e transferências de apoio informal. In: Lebrão ML, Duarte YAO, organizadores. SABE - Saúde, Bem-estar e Envelhecimento - o projeto SABE no município de São Paulo: uma abordagem inicial. Brasília: Organização Pan-Americana da Saúde; 2003. p. 201-24.

27. Lin N, Ye X, Ensel WM. Social support and depressed mood: a structural analysis. J Health Soc Behav 1999; 40:344-59.

Recebido em 01/Nov/2006

Versão final reapresentada em 12/Abr/2007

Aprovado em 16/Mai/2007 